\title{
Purification and Characterization of NF-R2 that Regulates the Expression of the Human Multidrug Resistance (MDR1) Gene
}

\author{
Toshiro Takatori, Mitsuo Ogura ${ }^{1}$ and Takashi Tsuruo ${ }^{2}$ \\ Institute of Applied Microbiology, The University of Tokyo, Yayoi 1-1-1, Bunkyo-ku, Tokyo 113, and \\ Cancer Chemotherapy Center, Kami-Ikebukuro 1-37-1, Toshima-ku, Tokyo 170
}

\begin{abstract}
NF-R2 is a DNA-binding protein that interacts with the MDR1 gene proximal promoter sequence. We previously reported that NF-R2 binds within the promoter's -126 and -102 regions, which contain the ATTCAGTCA motif. In the present study, we have purified NF-R2 from the nuclear extract of K562/ADM cells, a multidrug-resistant cell line derived from human myelogenous leukemia K562 cells, using sequential chromatography on Sephacryl S-300, DEAE-Sepharose, heparin-Sepharose and a DNA affinity column consisting of a repetitive synthetic ATTCAGTCA motif coupled to Sepharose. NF-R2 runs as a single protein of $75 \mathrm{kDa}$ on SDS-PAGE (sodium dodecyl sulfate-polyacrylamide gel electrophoresis). CAT (chloramphenicol acetyltransferase) expression assay and gel mobility shift competition assay with mutated promoters revealed that the ATTCAGTCA motif is a positive regulatory element of MDR1 gene and that the motif is important for NF-R2 binding. These results suggest that NF-R2 may be involved in the positive regulation of the MDR1 gene transcription.
\end{abstract}

Key words: Multidrug resistance - MDR 1 gene - Promoter - DNA-binding protein

Multidrug resistance (MDR) is a major problem in cancer chemotherapy. ${ }^{1)}$ MDR is a phenomenon in which tumor cells exposed to several anti-tumor agents, such as vincristine, adriamycin or actinomycin $\mathrm{D}$, become resistant to those agents, as well as to a variety of other chemically and structurally diverse chemotherapeutic agents. ${ }^{2)}$ This resistance results from the expression of the MDR1 gene, which encodes a protein, termed P-glycoprotein, that acts as an active efflux pump to exclude the antitumor agents from the cells. ${ }^{3}$ MDR1 mRNA is expressed at substantial levels in many MDR cell lines, ${ }^{4,5)}$ and it has been suggested that the transcriptional activation of MDR1 gene is important during the MDR development. ${ }^{6,7)}$

We previously demonstrated ${ }^{8)}$ that the upstream region of the MDR1 gene, at -131 to +10 relative to the major transcription initiation site $(+1),{ }^{9)}$ showed promoter activity. The activity was determined by the ability of this region to direct the expression of the chloramphenicol acetyltransferase (CAT) gene upon transfection into K562/ADM cells, an adriamycinresistant subline of human myelogenous leukemia K562 cells. $^{10)}$ In K562/ADM nuclear extract, we detected three DNA-binding proteins, termed NF-R1, -R2, and $-\mathrm{R} 3$, which could interact with the promoter sequence. ${ }^{8}$ ) NF-R1 has been purified and characterized as being related to the negative regulation of the MDR1 gene

\footnotetext{
${ }^{1}$ Present address: School of Marine Science and Technology, Tokai University, Orido 3-20-1, Shimizu-shi, Shizuoka 424.

${ }^{2}$ To whom correspondence should be addressed.
}

transcription. ${ }^{11)}$ This protein could bind to both the ATTCAGTCA motif $(-119$ to -111$)$ and the GC-box motif $(-52$ to -41$)$. NF-R2 and NF-R 3 could bind to the sequence containing the ATTCAGTCA motif and the GC-box motif, respectively. However, these two factors have not been well characterized as yet.

In our previous paper, ${ }^{8)}$ we showed that the deletion of -131 to -100 , which contained the ATTCAGTCA motif, reduced the promoter activity to $20 \%$ that of the intact sequence, whereas the deletion of -66 to -37 , which contained the GC-box, reduced the activity to $50 \%$. Therefore we considered that the protein binding to the ATTCAGTCA motif played an important role in MDR1 gene transcription. NF-R2 could bind to the sequence and is a candidate for a regulator of the gene.

Here we report the purification of NF-R2 using sequence-specific DNA affinity chromatography. We characterize NF-R2 as a trans-acting factor involved in the MDR1 transcription in K562/ADM cells by means of gel mobility shift competition analysis and CAT expression assay.

\section{MATERIALS AND METHODS}

Cell culture K562/ADM, which was established in our laboratory, ${ }^{10)}$ was grown in RPMI 1640 medium (Nissui Co., Ltd.) supplemented with $5 \%$ fetal bovine serum and $100 \mu \mathrm{g} / \mathrm{ml}$ kanamycin.

Purification of NF-R2 Nuclear extract of K562/ADM cells was prepared as described previously ${ }^{8)}$ The crude nuclear extract was dialyzed against TM buffer ${ }^{12)}$ (50 
$\mathrm{m} M$ Tris- $\mathrm{HCl}, \mathrm{pH} 7.9,12.5 \mathrm{~m} M \mathrm{MgCl}_{2}, 1 \mathrm{~m} M$ EDTA, $1 \mathrm{~m} M$ DTT and $20 \%$ ethylene glycol) containing $0.2 M$ $\mathrm{KCl}$ and fractionated by Sephacryl S-300 column chromatography. NF-R2 activity was determined by gel mobility shift assay using a ${ }^{32} \mathrm{P}-$ TaqI-HaeII fragment as a probe. Fractions containing NF-R2, which eluted at $65 \%$ of column volume, were pooled, diluted to a concentration of $0.1 M \mathrm{KCl}$ with TM buffer, and applied to a DEAE-Sepharose column. The flow-through fraction containing NF-R2 was loaded onto a heparin-Sepharose column. The column was washed with $0.1 \mathrm{M} \mathrm{KCl-TM}$ buffer and eluted with $0.2 \mathrm{M} \mathrm{KCl-TM}$ buffer. The eluate was dialyzed against NB buffer ${ }^{13)}(20 \mathrm{~m} M$ Tris- $\mathrm{HCl}, \mathrm{pH}$ $7.9,2.5 \mathrm{mM} \mathrm{MgCl} 2,1 \mathrm{~m} M$ EDTA, 5\% ethylene glycol) containing $50 \mathrm{mM} \mathrm{KCl}$ and applied to the DNA affinity column coupled with a polymerized 46mer DNA fragment $(-126$ to -81$)$ containing the ATTCAGTCA motif, which was prepared according to the method described by Kadonaga and Tjian. ${ }^{13)}$ This column was eluted with a stepwise $\mathrm{KCl}$ gradient from $0.1 M$ to $1 M$ containing NB buffer. The $0.3 \mathrm{M} \mathrm{KCl}-\mathrm{NB}$ buffer eluate containing NF-R2 was diluted with NB buffer to give $50 \mathrm{mM} \mathrm{KCl}$, reapplied to the DNA affinity column and purified as above. Purified NF-R2 was analyzed by using SDS-PAGE, ${ }^{14)}$ with a $4-20 \%$ gradient polyacrylamide gel, and visualized by silver staining. ${ }^{15)}$

Gel mobility shift assay The probe for detecting NF-R2 activity was prepared as follows. A pUM8 plasmid $^{8)}$ was digested with TaqI and HaeII to obtain the fragment $(-130$ to -56$)$ which was end-labeled with $\left[\alpha-{ }^{32} \mathrm{P}\right]$ dCTP (3000 Ci/mmol; Amersham) using the Klenow fragment of DNA polymerase I. The binding reactions and the subsequent procedures have been described previously. ${ }^{8)}$ The final concentration of $\mathrm{KCl}$ in the binding reaction was adjusted to $90 \mathrm{mM}$. Carrier DNA was not added in the assay for the fractions of the second cycle of the DNA affinity column.

Southwestern blot analysis A $20 \mu 1$ aliquot of the purified fraction mixed with $5 \mu 1$ of $5 \times$ SDS-PAGE buffer $^{8)}$ was subjected to SDS-PAGE using a $4-20 \%$ gradient polyacrylamide gel. The southwestern blot procedure was performed as described previously ${ }^{8)}$ using a ${ }^{32} \mathrm{P}-\mathrm{Taq}$-HaeII fragment as a probe.

Plasmids Oligonucleotides for preparation of CAT construct plasmids or competition analysis were synthesized with a DNA synthesizer (Applied Biosystems 380B). CAT construct plasmids were prepared as follows. Oligonucleotides, wild-type (WT), $\Delta(-122$ to -116$)$ and $\Delta(-115$ to -109$)$ (listed in Fig. 4) possessing cohesive ends complementary to $\mathrm{XmaI}$ and $\mathrm{BamHI}$ at each end were prepared. These oligonucleotides were cloned between the XmaI and BamHI sites of p99MDRCAT ${ }^{8)}$ a CAT construct plasmid plasmid in which a part of the MDR1 promoter $(-99$ to +9$)$ is positioned upstream of the CAT reporter gene, and three plasmids, pWT (Bam), p $\Delta(-122$ to -116$)$ and $\mathrm{p} \Delta(-115$ to -109 ), were obtained, respectively. These plasmids possessed an artificial BamHI site at -99 . pWT (Bam) showed reduced promoter activity (70\%, data not shown) compared with the intact MDR1 promoter, p131MDR-CAT. ${ }^{8)}$ WT was end-labeled with $\left[\alpha-{ }^{32}\right.$ P $]$ dCTP using a Klenow fragment of DNA polymerase I for the gel mobility shift competition assay.

CAT expression assay Transfection and assays of CAT were performed as described previously. ${ }^{8,11)}$ In short, CAT constructs $(20 \mu \mathrm{g})$ with pSV- $\beta$-gal $(20 \mu \mathrm{g}$; Promega) were transfected into $\mathrm{K} 562 / \mathrm{ADM}$ cells $\left(2 \times 10^{7}\right.$ cells $)$ by electroporation (Bio-Rad; Gene Pulser). At $48 \mathrm{~h}$ after transfection, cells were harvested and lysed by four cycles of freezing-thawing $\left(-70^{\circ} \mathrm{C}\right.$ and $\left.37^{\circ} \mathrm{C}\right)$. The cell lysates were centrifuged, and the supernatants were assayed for $\mathrm{CAT}^{16)}$ and $\beta$-galactosidase activities. ${ }^{17)} \beta$ Galactosidase activity served as an internal standard to correct for differences in transfection efficiency.

\section{RESULTS}

Purification of NF-R2 We previously detected NF-R1 and NF-R2, which bound to the sequence containing the ATTCAGTCA motif (Fig. 1), in the K562/ADM nuclear extract. ${ }^{11)}$ NF-R2 was separated from NF-R1 by Sephacryl S-300 column chromatography. ${ }^{11)}$ NF-R2 has been purified from this fraction according to the scheme shown in Fig. 2. The NF-R2 activity was measured by gel mobility shift assay. The NF-R2 activity passed through the DEAE-Sepharose column but bound to the heparinSepharose column. The bound NF-R2 was eluted with $0.2 M \mathrm{KCl}-\mathrm{TM}$ buffer. Next, the ATTCAGTCA motifspecific DNA affinity column ${ }^{11)}$ was employed. Among the column fractions, the NF-R2 activity was most prom-

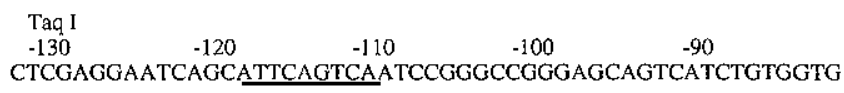

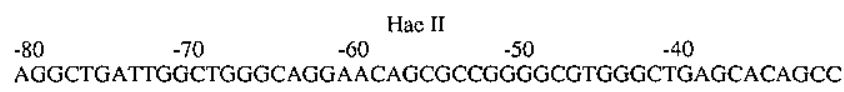

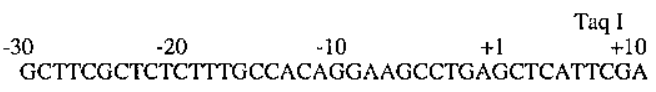

Fig. 1. Structure of the proximal promoter region of MDR1. The nucleotide sequence of the MDR1 promoter is shown from -133 to $+10(+1$ : transcription start site $) .{ }^{9)}$ The ATTCAGTCA motif is underlined. Several recognition sites of restriction enzymes are shown above the sequence. 


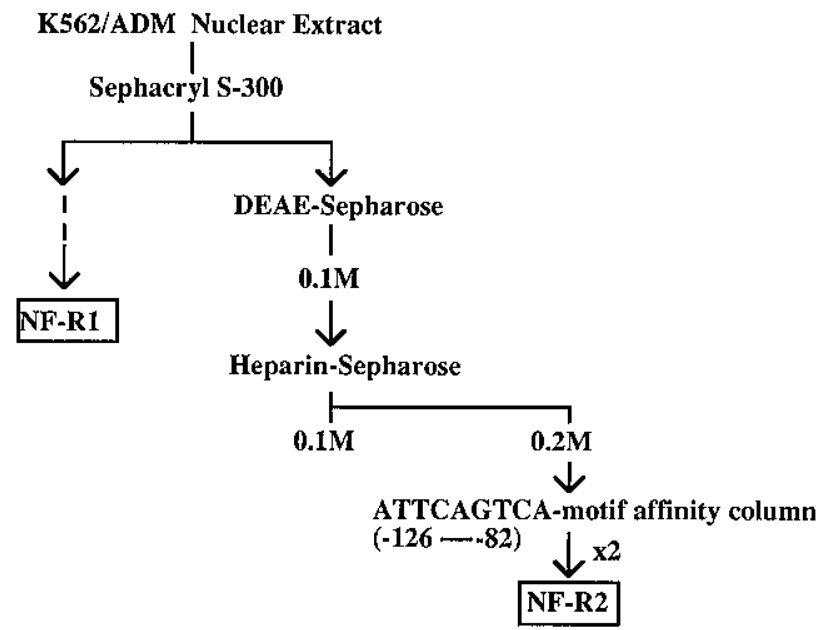

Fig. 2. Schematic representation of the purification procedure of NF-R2. The K562/ADM nuclear extract was fractionated according to this scheme to purify NF-R2. The molar number indicates the concentration of $\mathrm{KCl}$ in the buffer.
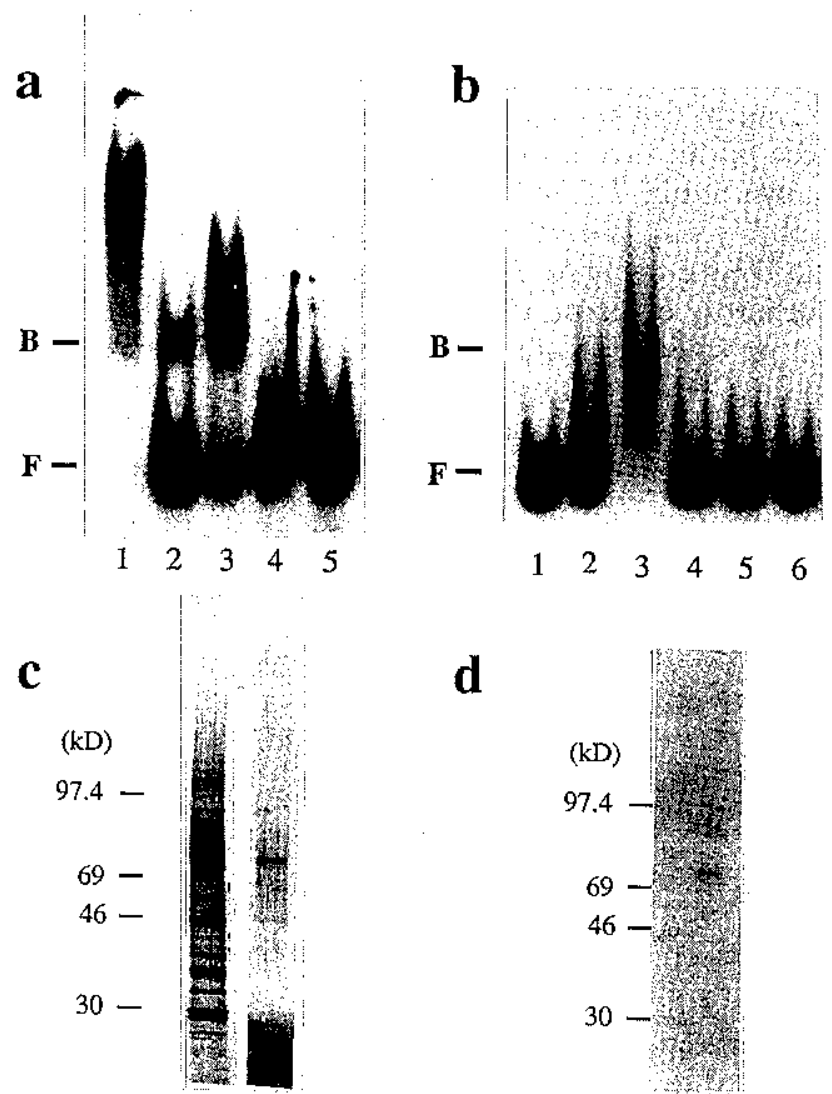

d

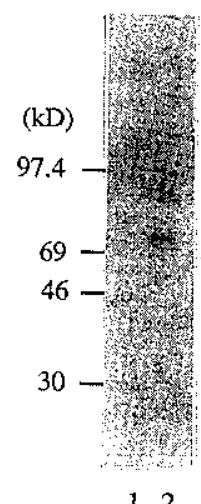

inent in $0.3 \mathrm{M} \mathrm{KCl}$ eluate (Fig. 3a). As the fraction still contained some other proteins, it was subjected to a second cycle on the column (Fig. 3b). Finally NF-R2 having a molecular size of $75 \mathrm{kDa}$ on SDS-PAGE (Fig. $3 c$ ) was obtained, although a minor band of $46 \mathrm{kDa}$ was still detected. The $75 \mathrm{kDa}$ protein showed a DNAbinding activity on southwestern blot analysis (Fig. 3d). Importance of ATTCAGTCA motif for NF-R2 binding We previously found that NF-R2 specifically bound to the sequence which consisted of 25 bp containing the ATTCAGTCA motif $(-126$ to -102$)$. In order to examine the binding specificity of the factor in more detail, we prepared three oligonucleotides (Fig. 4a) and performed a competition analysis in gel mobility shift assay. WT is a $33 \mathrm{bp}$ oligonucleotide corresponding to -132 to -100 , which contains the ATTCAGTCA motif. The other two are deleted mutants of WT, each with a 7 bp deletion, AGCATTC $(-122$ to -116$)$ and AGTCAAT $(-115$ to -109$)$. These mutants were termed $\Delta(-122$ to -116$)$ and $\Delta(-115$ to -109$)$, respectively.

The formation of the ${ }^{32}$ P-WT-NF-R2 complex was inhibited by the presence of increasing quantities of cold WT, whereas the mutants inhibited it very weakly (Fig. 4b). This result indicated that the deleted sequence was important for binding to NF-R2. In order to examine more precisely the importance of the ATTCAGTCA motif for NF-R2 binding, we performed another competition analysis where oligonucleotides carrying two base changes in the ATTCAGTCA motif $(-119$ to -111$)$ (Fig. 5) were used. According to this competition experiment, CM3, with a GC to TG $(-121,-120)$ conversion, seemed to have similar affinity for NF-R2 to the WT oligonucleotide. CM5, CM7 and CM8 had TC to

Fig. 3. Purification of NF-R2 from K562/ADM nuclear extract. (a) The $0.2 \mathrm{M}$ KCl-TM buffer eluate of the heparinSepharose column was applied to the DNA affinity column (first cycle). The DNA affinity column fractions were subject to the gel mobility shift assay. The ${ }^{32} \mathrm{P}-$ TaqI-HaeII fragment was used as a probe. Lane 1, input; lane 2, flow-through; lane $3,0.3 \mathrm{M} \mathrm{KCl}$ eluate; lane $4,0.5 \mathrm{M} \mathrm{KCl}$ eluate; lane $5,0.7 \mathrm{M}$ $\mathrm{KCl}$ eluate. $\mathrm{B}$ and $\mathrm{F}$ indicate the probe-protein complex and the free probe, respectively. (b) The $0.3 \mathrm{M} \mathrm{KCl}$ eluate of the first cycle was again applied to the affinity column (second cycle) and assayed as above. Lane 1, flow-through; lane 2, 0.2 $M \mathrm{KCl}$ eluate; lane $3,0.3 M \mathrm{KCl}$ eluate; lane $4,0.4 M \mathrm{KCl}$ eluate; lane $5,0.5 \mathrm{M} \mathrm{KCl}$ eluate; lane $6,0.6 \mathrm{M} \mathrm{KCl}$ eluate. (c) SDS-polyacrylamide gel electrophoresis. The $0.3 M$ eluate of the first cycle (Input) and the second cycle (Eluate) of the affinity column were loaded on a $4-20 \%$ gradient polyacrylamide-SDS gel, and polypeptides were visualized by silver staining. (d) Southwestern blot. The flow-through (lane 1) and the $0.3 M$ eluate (lane 2 ) of the second cycle was subjected to the southwestern blot analysis. ${ }^{32} \mathrm{P}-$ TaqI-HaelI was used as a probe. 
a

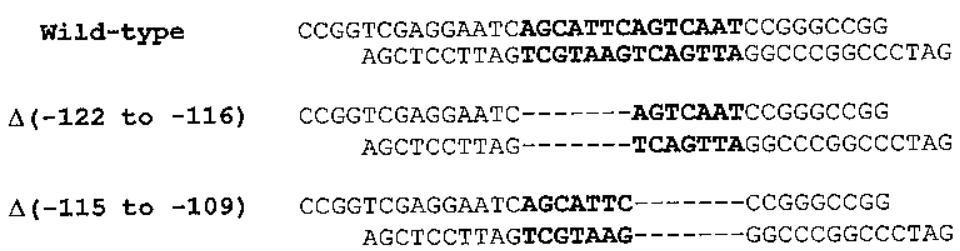

b

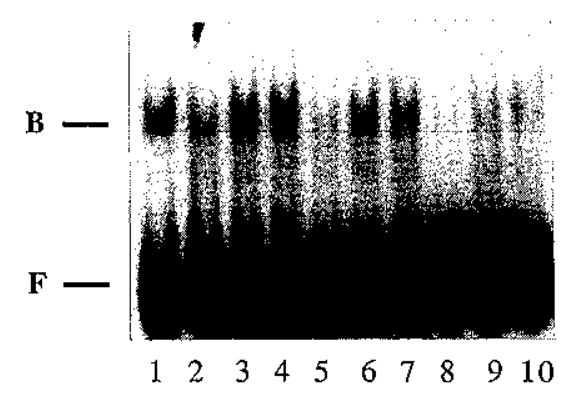

Fig. 4. Functional analysis of NF-R2 using deleted mutants. (a) The nucleotides used in the analysis are shown. Wild-type (WT) is a 33-bp oligonucleotide corresponding to -132 to -100 relative to the major transcription initiation site of MDR1 gene. $\Delta(-122$ to -116$)$ and $\Delta(-115$ to -109$)$ are internally deleted sequences of WT. These three oligonucleotides possess cohesive ends complementary to XmaI (CCGG) and BamHI (CTAG) sites at each end. (b) Gel mobility shift competition analysis. The NF-R2 fraction was incubated with ${ }^{32} \mathrm{P}-\mathrm{WT}$ at $4^{\circ} \mathrm{C}$ and subjected to the PAGE (4\% polyacrylamide). Competitors were added at $0 \mathrm{pmol}$ (lane 1), 0.5 pmol $(2,3,4) 1.0$ pmol $(5,6,7), 2.0$ pmol $(8$, $9,10)$. Competitors: $2,5,8$; WT, $3,6,9 ; \Delta(-122$ to -116$)$; $4,7,10, \Delta(-115$ to -109$)$. $B$ and $F$ indicate the probeprotein complex and the free probe, respectively. (c) CAT expression assay. $\mathrm{pWT}, \mathrm{p} \Delta(-122$ to -116$)$ and $\mathrm{p} \Delta(-115$ to -109) were cotransfected with $\mathrm{pSV}-\beta$-gal into K562/ADM cells, and $48 \mathrm{~h}$ later the cell extracts were prepared and analyzed as described in "Materials and Methods." The acetylated chloramphenicol was quantified by using a Bio-image analyzer BA-100 (Fuji Photo Film Co.) to determine the level of CAT expression of mutated plasmids relative to WT. The average and the standard deviation of three independent experiments are presented.

AG $(-117,-116)$, TC to GA $(-113,-112)$ and AA to $\mathrm{CC}(-111,-110)$ conversions, respectively, and showed lower affinity for NF-R2 than the WT oligonucleotide. CM4 and CM6, with AT to TA $(-119,-118)$, AG to TT $(-115,-114)$ conversion, respectively showed the lowest affinity for NF-R2. Oligonucleotide carrying the GC-box motif could not compete with ${ }^{32} \mathrm{P}$-WT for binding to NF-R2. These observations showed that the ATTCAGTCA motif was important for NF-R2 binding. Functional analysis of deletion mutants To provide further evidence of the role of NF-R2, we examined the ability of the deletion mutants used above (Fig. 4a) to support transcription in K562/ADM cells when linked to the CAT reporter gene. Three CAT expression plasmids, pWT (Bam), $\mathrm{p} \Delta(-122$ to -116$)$ and $\mathrm{p} \Delta(-115$

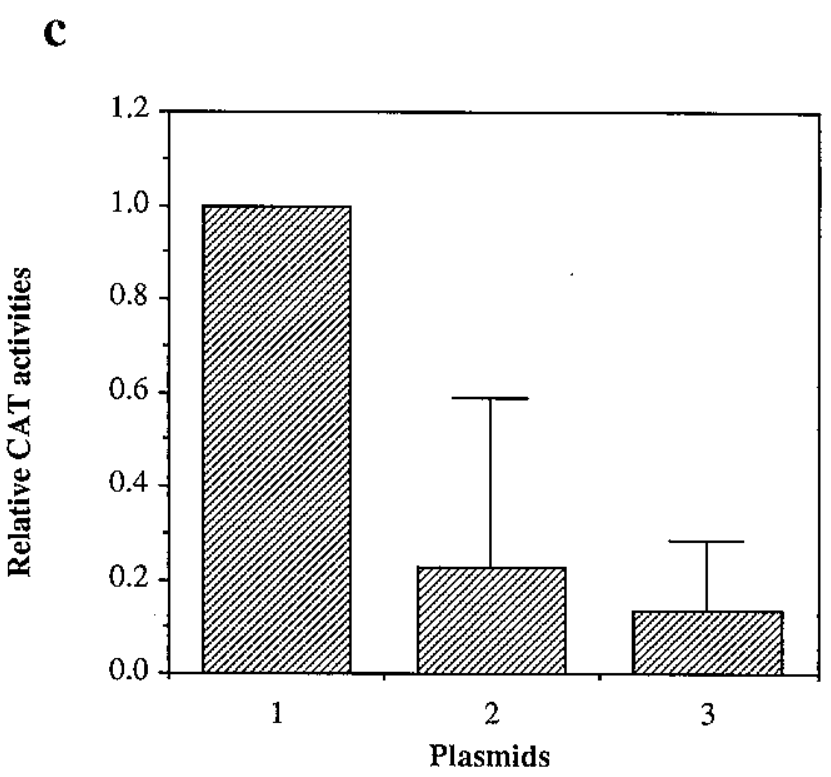

to -109 ), prepared as described in "Materials and Methods," were introduced into K562/ADM cells, and $48 \mathrm{~h}$ later, the cell lysates were tested for CAT activity. As shown in Fig. 4c, both $\mathrm{p} \Delta(-122$ to -116$)$ and $\mathrm{p} \Delta(-115$ to -109$)$ showed low levels of the promoter activity (20\% of pWT (Bam)), suggesting the ATTCAGTCA motif is a cis-element acting positively on the MDR1 transcription.

\section{DISCUSSION}

We have described the purification of NF-R2 from K562/ADM nuclear extract using four sequential column chromatographies. The gel-filtered nuclear extract of K562/ADM was applied to a DEAE-Sepharose 
a

wild-type
CM3
CM4
CM5
CM6
CM7
CM8
GC-box
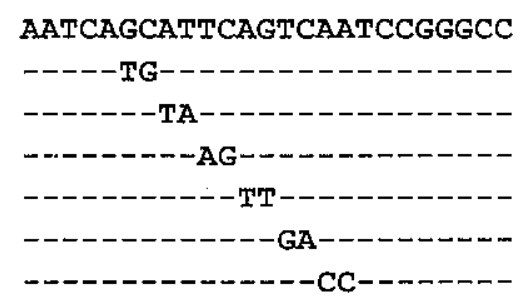

AGGAACAGCGCCGGGGCGTGGGCTGA

b

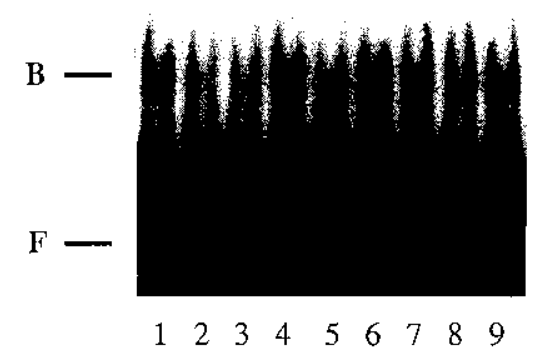

Fig. 5. Base-change effect on the binding of NF-R2. (a) Oligonucleotides used in this assay are shown. The mutants which have two base changes in the ATTCAGTCA-motif (CM3-8 ${ }^{11)}$ ) were used as competitors. The GC-box $(-64$ to -39) was used as a non specific competitor. (b) Gel mobility shift competition analysis. The NF-R2 fraction was incubated with ${ }^{32} \mathrm{P}$-WT at $4^{\circ} \mathrm{C}$ in the absence or presence of competitors shown in (a) and subjected to the PAGE (4\% polyacrylamide). The competitors were added at $1.2 \mathrm{pmol}$ each. In lane 1 , no competitor was added. Lane 2, wild-type; lanes 3-8, CM3-8, respectively; lane 9, GC-box. $\mathbf{B}$ and $F$ indicate the probe-protein complex and the free probe, respectively.

column and a heparin-Sepharose column, followed by two cycles of the DNA affinity column. During these procedures, NF-R2 was purified as a single peptide of $75 \mathrm{kDa}$, as shown by the SDS-PAGE pattern (Fig. 3c). Southwestern blot analysis showed that the purified protein possessed the ability to bind DNA (Fig. 3d). On southwestern blot analysis using $0.2 \mathrm{M}$ eluate from heparin-Sepharose column, we also detected the same protein as the only protein that bound to the ATTCAGTCA motif (data not shown).

The competition experiments of gel mobility shift assay showed that the ATTCAGTCA motif was important for NF-R2 binding, and two base-changes of the motif did not greatly affect the NF-R2 binding ability (Fig. 5). On the other hand, these changes did affect the NF-R1 binding ability. ${ }^{11)}$ CAT expression assay showed that two base-changes of the ATTCAGTCA motif which abolished the NF-R1 binding ability resulted in the enhancement of CAT expression. ${ }^{15)}$ Thus, we considered that NF-R1 has a role in negative regulation. The ATTCAGTCA motif itself is a positive element of the transcription (Fig. 4b, c). Therefore it is likely that NF$\mathrm{R} 2$ contributes to the positive regulation of the MDR1 gene transcription. To examine the role of NF-R2, it is useful to exclude the effect of NF-R1. Among the four mutants, CM5-8, which had lost NF-R1 binding ability, ${ }^{11)}$ CM6 mutant, which had relatively low affinity for NF-R2, showed low CAT activity compared with the others(CM5, 7, 8). ${ }^{11)}$ The ATTCAGTCA motif could bind to NF-R1 and -R2. Therefore it is difficult to establish clearly the character of these factors by such in vivo expression assays. Molecular cloning of these factors could help to analyze more precisely their roles in the transcription.

We have shown that NF-R2 could bind to the ATTCAGTCA motif and that this binding could not be inhibited by the GC-box motif, while NF-R1 could bind to both motifs at the same site of the protein. These results suggest that NF-R2 is distinct from NF-R1 with respect to the binding specificity as well as molecular size (NF-R1 has a molecular weight of $110 \mathrm{kDa}$ ). ${ }^{11)}$

We have shown that NF-RI bound to one strand of the duplex DNA on both ATTCAGTCA motif and GC-box motif. ${ }^{11)} \mathrm{Spl}$ and TFIIIA can also bind to one strand of the duplex DNA and could provide binding spaces for other proteins. ${ }^{18,19)}$ We have observed that both NF-R1 and NF-R2 could bind to the ATTCAGTCA motif concurrently (data not shown). Like Spl and TFIIIA, NF-R1 might bind to one strand of DNA to generate space for the cooperative binding of NF-R2.

Recently Kohno et al. have reported that two upstream regions, $(-258$ to -198$)$ and $(-136$ to -76$)$, were responsible for the activation of the MDR 1 gene in response to serum starvation or anticancer agents and heat shock, respectively, using KB cell lines. ${ }^{20,21)}$ It is unclear whether the ATTCAGTCA motif is responsible for the expression of the MDR1 gene in response to environmental stressess. It seems possible that NF-R 1 or $-\mathrm{R} 2$ is involved in these responses by interacting with some stress proteins.

\section{ACKNOWLEDGMENTS}

We thank Dr. A. Shiraishi for helpful suggestions about the manuscript. We also thank Drs. Y. Sugimoto and M. Naito for helpful discussions.

(Received October 7, 1992/Accepted November 30, 1992) 


\section{REFERENCES}

1) Bradley, G., Juranka, P. F. and Ling, V. Mechanism of multidrug resistance. Biochim. Biophys. Acta, 948, 87-128 (1988).

2) Bech-Hansen, N. T., Till, J. E. and Ling, V. Pleiotropic phenotype of colchicine-resistant $\mathrm{CHO}$ cells: crossresistance and collateral sensitivity. J. Cell. Physiol., 88, 23-31 (1976).

3) Gottesman, M. M. and Pastan, I. The multidrug transporter, a double-edged sword. J. Biol. Chem., 263, 1216312166 (1988).

4) Roninson, I. B., Chin, J. E., Choi, K., Gros, P., Housman, D. E., Fojo, A., Shen, D.-w., Gottesman, M. M. and Pastan, I. Isolation of human mdr DNA sequences amplified in multidrug-resistant KB carcinoma cells. Proc. Natl. Acad. Sci. USA, 83, 4538-4542 (1986).

5) Shen, D.-w., Fojo, A., Chin, J. E., Roninson, I. B., Richart, N., Pastan, I. and Gottesman, M. M. Human multidrug-resistant cell lines: increased mdrl expression can precede gene amplification. Science, 232, 643-645 (1986).

6) Fojo, A. T., Ueda, K., Slamon, D. J., Poplack, D. G., Gottesman, M. M. and Pastan, I. Expression of a multidrug-resistance gene in human tumors and tissues. Proc. Natl. Acad. Sci. USA, 84, 265-269 (1987).

7) Bourhis, J., Benard, J., Hartman, O., Boccon-Gibod, L., Lemerle, $J$. and Riou, G. Correlation of MDR1 gene expression with chemotherapy in neuroblastoma. J. Natl. Cancer Inst., 81, 1401-1405 (1989).

8) Ogura, M., Takatori, T., Sugimoto, Y., and Tsuruo, T. Identification and characterization of three DNA-binding proteins on the promoter of the human $M D R 1$ gene in drug-sensitive and -resistant cells. Jpn. J. Cancer Res., 82, 1151-1159 (1991).

9) Ueda, K., Pastan, I. and Gottesman, M. M. Isolation and sequence of the promoter gegion of the human multidrugresistance (P-glycoprotein) gene. J. Biol. Chem., 262, 17432-17436 (1987).

10) Tsuruo, T., Iida-Saito, H., Kawabata, H., Oh-hara, T., Hamada, $\mathbf{H}$. and Utakoji, T. Characteristics of resistance to adriamycin in human myelogenous leukemia K562 resistant to adriamycin and in isolated clones. Jpn. J. Cancer
Res., 77, 682-692 (1986).

11) Ogura, M., Takatori, T. and Tsuruo, T. Purification and characterization of NF-R1 that regulates the expression of the human multidrug resistance (MDR1) gene. Nucleic Acids Res, 20, $5811-5817$ (1992).

12) Briggs, M. R., Kadonaga, J. T., Bell, S. P. and Tjian, R. Purification and biochemical characterization of the promoter-specific transcription factor, Spl. Science, 234, 47-52 (1986).

13) Kadonaga, J. T. and Tjian, R. Affinity purification of sequence-specific DNA binding proteins. Proc. Natl. Acad. Sci. USA, 83, 5889-5893 (1986).

14) Laemmli, U. K. Cleavage of structural proteins during the assembly of the head of bacteriophage T4. Nature, 227, 680-685 (1970).

15) Ohsawa, K. and Ebata, N. Silver stain for detecting 10femtogram quantities of protein after polyacrylamide gel electrophoresis. Anal. Biochem., 135, 409-415 (1983).

16) Gorman, C. M., Moffat, L. F. and Howard, B. H. Recombinant genomes which express chloramphenicol acetyltransferase in mammalian cells. Mol. Cell. Biol., 2, 1044-1051 (1982).

17) Rosental, N. Identification of regulatory elements of cloned genes with functional assays. Methods Enzymol., 152, 704-720 (1987).

18) Gidoni, D., Dynan, S. W. and Tjian, R. Bidirectional SV40 transcription mediated by tandem Spl binding interactions. Nature, 312, 409-413 (1984).

19) Sakonju, S. and Brown, D. D. Contact points between a positive transcription factor and Xenopus 5S RNA gene. Cell, 31, 395-405 (1982).

20) Tanimura, H., Kohno, K., Sato, S., Uchiumi, T., Miyazaki, M., Kobayashi, M. and Kuwano, M. The human multidrug resistance 1 promoter has an element that responds to serum starvation. Biochem. Biophys. Res. Commun., 183, 917-924 (1992).

21) Miyazaki, M., Kohno, K., Uchiumi, T., Tanimura, H., Matsuo, K., Nasu, M. and Kuwano, M. Activation of human multidrug resistance-1 gene promoter in response to heat shock stress. Biochem. Biophys. Res. Commun., 187, 677-684 (1992). 